TRANSACTIONS OF THE

AMERICAN MATHEMATICAL SOCIETY

Volume 358, Number 5 , Pages 2259-2268

S 0002-9947(05)03831-6

Article electronically published on October 31,2005

\title{
THE FOURTH POWER MOMENT OF AUTOMORPHIC $L$-FUNCTIONS FOR $G L(2)$ OVER A SHORT INTERVAL
}

\author{
YANGBO YE
}

\begin{abstract}
In this paper we will prove bounds for the fourth power moment in the $t$ aspect over a short interval of automorphic $L$-functions $L(s, g)$ for $G L(2)$ on the central critical line $\operatorname{Re} s=1 / 2$. Here $g$ is a fixed holomorphic or Maass Hecke eigenform for the modular group $S L_{2}(\mathbb{Z})$, or in certain cases, for the Hecke congruence subgroup $\Gamma_{0}(\mathcal{N})$ with $\mathcal{N}>1$. The short interval is from a large $K$ to $K+K^{103 / 135+\varepsilon}$. The proof is based on an estimate in the proof of subconvexity bounds for Rankin-Selberg $L$-function for Maass forms by Jianya Liu and Yangbo Ye (2002) and Yuk-Kam Lau, Jianya Liu, and Yangbo Ye (2004), which in turn relies on the Kuznetsov formula (1981) and bounds for shifted convolution sums of Fourier coefficients of a cusp form proved by Sarnak (2001) and by Lau, Liu, and Ye (2004).
\end{abstract}

\section{Introduction}

For the Riemann zeta function and Dirichlet $L$-functions, estimates for their power moments on the critical line $\operatorname{Re} s=1 / 2$ played central roles in analytic number theory. Classical results on short intervals

$$
\int_{K}^{K+K^{\alpha}}\left|\zeta\left(\frac{1}{2}+i t\right)\right|^{4} d t \ll K^{\alpha+\varepsilon}
$$

were proved for $\alpha=7 / 8$ by Heath-Brown [9] and for 2/3 by Iwaniec [11, for any $\varepsilon>0$. In this paper, we want to prove a similar result for automorphic $L$-functions attached to a certain holomorphic or Maass cusp form $g$ for $\Gamma_{0}(\mathcal{N})$.

To describe our results, we need bounds towards the Ramanujan conjecture for Maass forms. In terms of representation theory, let $\pi$ be an automorphic cuspidal representation of $G L_{2}\left(\mathbb{Q}_{A}\right)$ with unitary central character and local Hecke eigenvalues $\alpha_{\pi}^{(j)}(p)$ for $p<\infty$ and $\mu_{\pi}^{(j)}(\infty)$ for $p=\infty, j=1,2$. Then bounds toward the Ramanujan conjecture are

$$
\begin{aligned}
\left|\alpha_{\pi}^{(j)}(p)\right| \leq p^{\theta} \quad & \text { for } p \text { at which } \pi \text { is unramified, } \\
\left|\operatorname{Re}\left(\mu_{\pi}^{(j)}(\infty)\right)\right| \leq \theta & \text { if } \pi \text { is unramified at } \infty .
\end{aligned}
$$

Received by the editors March 26, 2004 and, in revised form, July 27, 2004.

2000 Mathematics Subject Classification. Primary 11F66.

This project was sponsored by the National Security Agency under Grant Number MDA90403-1-0066. The United States Government is authorized to reproduce and distribute reprints notwithstanding any copyright notation herein.

(C)2005 American Mathematical Society
ublic domain 28 years from publication Reverts to public domain 28 years from publication 
These bounds were proved for $\theta=1 / 4$ by Selberg and Kuznetsov [16, for $\theta=1 / 5$ by Shahidi 23] and Luo, Rudnick, and Sarnak [20, for $\theta=1 / 9$ by Kim and Shahidi [14, and most recently for $\theta=7 / 64$ by Kim and Sarnak [13].

The automorphic $L$-functions we will consider are

$$
\begin{aligned}
L(s, g) & =\sum_{n \geq 1} \frac{\lambda_{g}(n)}{n^{s}} \\
& =\prod_{p \nmid \mathcal{N}}\left(1-\lambda_{g}(p) p^{-s}+p^{-2 s}\right)^{-1} \cdot \prod_{p \mid \mathcal{N}}\left(1-\lambda_{g}(p) p^{-s}\right)^{-1},
\end{aligned}
$$

where $g$ is a holomorphic or Maass cusp Hecke eigenform for $\Gamma_{0}(\mathcal{N})$, and its twist by a real primitive character $\chi$ modulo $\mathcal{Q}$ with $\mathcal{N} \mid \mathcal{Q}$ :

$$
L(s, g \otimes \chi)=\sum_{n \geq 1} \frac{\lambda_{g}(n) \chi(n)}{n^{s}}=\prod_{p \nmid \mathcal{Q}}\left(1-\chi(p) \lambda_{g}(p) p^{-s}+p^{-2 s}\right)^{-1}
$$

for Re $s>1$. Following the setting in Conrey and Iwaniec [2], we will assume that $\mathcal{Q}$ is odd and square-free, and $\chi$ is the real, primitive character modulo $\mathcal{Q}$, i.e., the Jacobi symbol, so that the twisted cusp form $g_{\chi}$ (see (2.1) and (2.2) below) is a cusp form for $\Gamma_{0}\left(\mathcal{N}^{2}\right)$. As pointed in [2], p. 1176, $g_{\chi}$ is primitive even if the Hecke eigenform $g$ itself is not primitive. Our results, nevertheless, are valid in other cases, as long as the twisted $L$-function $L(s, g \otimes \chi)$ has a standard functional equation as in (2.3) (cf. Atkin and Li 1]). In particular, our theorem below is valid for $L(s, g)$ when $\mathcal{N}=1$. We will assume that $g$ is self-contragredient. If $g$ is holomorphic, we denote its weight by $\ell$. If $g$ is Maass, we denote its Laplace eigenvalue by $1 / 4+\ell^{2}$.

Theorem 1.1. Let $g$ be a fixed self-contragredient holomorphic or Maass Hecke eigenform for $\Gamma_{0}(\mathcal{N})$, and let $\chi$ be a real, primitive character mod $\mathcal{Q}$ with $\mathcal{N} \mid \mathcal{Q}$. Then

$$
\int_{K}^{K+L}\left|L\left(\frac{1}{2}+i t, g \otimes \chi\right)\right|^{4} d t \ll_{\varepsilon, \mathcal{N}, g, \mathcal{Q}}(K L)^{1+\varepsilon}
$$

for $L=K^{1-1 /(4+2 \theta)+\varepsilon}$. Here $\theta$ is given by bounds toward the Ramanujan conjecture in (1.1), and we can take $\theta=7 / 64$ with $1-1 /(4+2 \theta)=103 / 135$.

A subconvexity bound for $L(s, g)$ in the $t$ aspect was deduced by Good for holomorphic cusp form $g$ in [6, 7], and [8], and by Meurman for Maass $g$ in [21]:

$$
L\left(\frac{1}{2}+i t, g\right) \ll_{g}(1+|t|)^{1 / 3} \log ^{5 / 6}(2+|t|) .
$$

The goal of the present paper is not an improvement to this subconvexity bound for $L(s, g)$. By a standard argument (cf. Ivic [10, p. 197) though, our Theorem 1.1 implies

$$
L\left(\frac{1}{2}+i t, g\right) \ll_{g}(1+|t|)^{1 / 2-1 /(16+8 \theta)+\varepsilon}=(1+|t|)^{119 / 270+\varepsilon} .
$$

Certainly our (1.3) is not as good as (1.2). Using (1.2), however, one can only get a fourth power moment bound of $\left(K^{4 / 3} L\right)^{1+\varepsilon}$, not as good as our Theorem 1.1.

Subconvexity bounds in the level $\mathcal{N}$ aspect and the $\ell$ aspect were studied extensively by Duke, Friedlander, and Iwaniec ([3], 4], [5]), and by Kowalski, Michel, and VanderKam [15. 
The proof of Theorem 1.1 is based on an argument in Jianya Liu and Yangbo Ye [18] and Yuk-Kam Lau, Jianya Liu, and Yangbo Ye [17]; see $\S 3$ below. In [18] subconvexity bounds for Rankin-Selberg $L$-functions $L(s, f \otimes g)$ were proved as the Laplace eigenvalue of the Maass cusp form $f$ goes to $\infty$, where $g$ is a fixed holomorphic or Maass cusp form. While the exponent $(3+2 \theta) / 4+\epsilon$ as claimed in [18 does not hold because of a gap in $\$ \S 4.14$ and 4.15 , the paper did prove a subconvexity bound

$$
L(1 / 2+i t, f \otimes g) \ll_{N, t, g, \epsilon} k^{(15+2 \theta) / 16+\epsilon}
$$

as pointed out in the first sentence in $\S 4.14$ (see Jianya Liu and Yangbo Ye [19]). In [17] (1.4) was improved to a better bound $O\left(k^{1-1 /(8+4 \theta)+\varepsilon}\right)$.

What was done in [18] and [17] was to express $L(1 / 2, f \otimes g)$ in terms of spectral decomposition of $f$ and $g$ by an approximate functional equation. Using the Kuznetsov trace formula ([16]) the spectral sum of $f$ is rewritten in terms of Kloosterman sums. Therefore the central value of $L(s, f \otimes g)$ is essentially expressed as a spectral sum of $g$ with Kloosterman sums as coefficients. An application of bounds for shifted convolution sums of Fourier coefficients of $g$ (Sarnak 22] with an improvement given in [17]) gives a subconvexity bound for $L(s, f \otimes g)$.

In this paper we will proceed to consider the continuous spectrum of the Laplacian in place of the Maass form $f$. This approach is motivated by Conrey and Iwaniec [2].

The author expresses his thanks to Chaohua Jia, Jianya Liu, and Wenzhi Luo for informative discussions, and to the referee for detailed, helpful comments and suggestions. Thanks also to the Obermann Center for Advanced Studies, the University of Iowa, for support services and nice academic environment.

\section{The APPRoXimate FUnCTIONAL EQUATION}

We know that the twisted $L$-function $L(s, g \otimes \chi)$ is entire, where $\chi$ is a real, primitive character modulo $\mathcal{Q}$ with $\mathcal{N} \mid \mathcal{Q}$. Note that $L(s, g \otimes \chi)$ is indeed the $L$ function attached to a twisted cusp form $g_{\chi}$. It is

$$
g_{\chi}(z)=\sum_{n \geq 1} n^{(k-1) / 2} \chi(n) \lambda_{g}(n) e(n z)
$$

when $g$ is holomorphic, and

$$
g_{\chi}(z)=y^{1 / 2} \sum_{n \neq 0} \chi(n) \lambda_{g}(n) K_{i l}(2 \pi|n| y) e(n x)
$$

when $g$ is Maass.

In any case, denote by

$$
\Lambda(s, g \otimes \chi)=L_{\infty}(s, g \otimes \chi) L(s, g \otimes \chi)
$$

the complete $L$-function, where

$$
L_{\infty}(s, g \otimes \chi)=\prod_{j=1}^{2} \Gamma_{\mathbb{R}}\left(s+\mu_{g_{\chi}}(j)\right)
$$

with $\Gamma_{\mathbb{R}}(s)=\pi^{-s / 2} \Gamma(s / 2)$. Here $\mu_{g_{\chi}}(1)$ and $\mu_{g_{\chi}}(2)$ are complex numbers associated to $g_{\chi}$ at $\infty$. According to Conrey and Iwaniec [2], p. 1188, our twisted cusp form 
$g_{\chi}$ satisfies the standard functional equation

$$
\Lambda(s, g \otimes \chi)=\varepsilon\left(s, g_{\chi}\right) \Lambda(1-s, g \otimes \chi),
$$

where $\varepsilon\left(s, g_{\chi}\right)=\tau\left(g_{\chi}\right) Q_{g_{\chi}}^{-s}$. Here $Q_{g_{\chi}}>0$ is the conductor of $g_{\chi}$ and $\tau\left(g_{\chi}\right) \in \mathbb{C}^{\times}$ satisfies $\tau\left(g_{\chi}\right) \tau\left(\tilde{g}_{\chi}\right)=Q_{g_{\chi}}$. Since $g$ is self-contragredient and $\chi$ is real, we have $\tau\left(g_{\chi}\right)^{2}=Q_{g_{\chi}}$.

We actually want a functional equation for a product of two such $L$-functions:

$$
L(s+i r, g \otimes \chi) L(s-i r, g \otimes \chi)=\gamma(s) L(1-s-i r, g \otimes \chi) L(1-s+i r, g \otimes \chi),
$$

where according to $(2.3)$

$$
\begin{aligned}
\gamma(s) & =\tau\left(g_{\chi}\right)^{2} Q_{g_{\chi}}^{-2 s} \prod_{j=1}^{2} \frac{\Gamma_{\mathbb{R}}\left(1-s+i r+\mu_{g_{\chi}}(j)\right) \Gamma_{\mathbb{R}}\left(1-s-i r+\mu_{g_{\chi}}(j)\right)}{\Gamma_{\mathbb{R}}\left(s+i r+\mu_{g_{\chi}}(j)\right) \Gamma_{\mathbb{R}}\left(s-i r+\mu_{g_{\chi}}(j)\right)} \\
& =\left(\frac{\pi^{2}}{Q_{g_{\chi}}}\right)^{2 s-1} \prod_{j=1}^{2} \frac{\Gamma\left(\left(1-s+i r+\mu_{g_{\chi}}(j)\right) / 2\right) \Gamma\left(\left(1-s-i r+\mu_{g_{\chi}}(j)\right) / 2\right)}{\Gamma\left(\left(s+i r+\mu_{g_{\chi}}(j)\right) / 2\right) \Gamma\left(\left(s-i r+\mu_{g_{\chi}}(j)\right) / 2\right)} .
\end{aligned}
$$

By Stirling's formula, we get (see similar computations in [22] and [18])

$$
\gamma(s)=\left(\frac{4 \pi^{2} / Q_{g_{\chi}}}{\left(\mu_{g_{\chi}}(1)+r^{2}\right)^{1 / 2}\left(\mu_{g_{\chi}}(2)+r^{2}\right)^{1 / 2}}\right)^{2 s-1}\left(1+\eta_{r}(s)\right),
$$

where the error term $\eta_{r}(s) \ll(1+|s|)^{3} /(1+|r|)$. We will consider the case of large $|r|$ with fixed $g$; hence $\gamma(s)$ is asymptotically $\left(Q_{g_{\chi}} r^{2} /\left(4 \pi^{2}\right)\right)^{1-2 s}$.

Following [22] and [18] again, we can express the central value of

$$
L(s+i r, g \otimes \chi) L(s-i r, g \otimes \chi)
$$

as

$$
\begin{aligned}
& L\left(\frac{1}{2}+i r, g \otimes \chi\right) L\left(\frac{1}{2}-i r, g \otimes \chi\right) \\
= & \frac{1}{\pi i} \int_{\operatorname{Re} s=2} X^{s} L\left(\frac{1}{2}+s+i r, g \otimes \chi\right) L\left(\frac{1}{2}+s-i r, g \otimes \chi\right) G(s) \frac{d s}{s} \\
& +O\left(\mid \int_{\operatorname{Re} s=2} X^{s} \eta_{r}\left(\frac{1}{2}-s\right) L\left(\frac{1}{2}+s+i r, g \otimes \chi\right)\right. \\
& \left.\times L\left(\frac{1}{2}+s-i r, g \otimes \chi\right) G(s) \frac{d s}{s} \mid\right),
\end{aligned}
$$

where

$$
X=\frac{Q_{g_{\chi}}}{4 \pi^{2}}\left(\mu_{g_{\chi}}(1)+r^{2}\right)^{1 / 2}\left(\mu_{g_{\chi}}(2)+r^{2}\right)^{1 / 2} .
$$

Here $G(s)$ is an analytic function in $-B \leq \operatorname{Re} s \leq B$ for a fixed $B>0$ satisfying

$$
G(0)=1, \quad G(s)=G(-s), \quad|G(s)| \ll(1+|s|)^{-A}
$$

for a fixed large constant $A$. We note that $X$ is real (and positive), because of our assumption on $g$ being self-contragredient.

We may shift the contour in the integral of the big $O$ term in (2.4) to Re $s=$ $1 / 2+\varepsilon$. This way $X^{s} \ll r^{1+\varepsilon}$. Recall that $\eta_{r}(1 / 2-s) \ll(1+|s|)^{3} /(1+|r|)$. Moreover,

$$
L\left(\frac{1}{2}+s+i r, g \otimes \chi\right) L\left(\frac{1}{2}+s-i r, g \otimes \chi\right) \ll_{\varepsilon, g} 1
$$


as $r \rightarrow \infty$, for $\operatorname{Re} s=1 / 2+\varepsilon$, because its Dirichlet series is absolutely convergent. All these show that the big $O$ term in $(2.4)$ is $\ll r^{\varepsilon}$.

To compute the main term in (2.4), we expand

$$
L(1 / 2+s+i r, g \otimes \chi) L(1 / 2+s-i r, g \otimes \chi)
$$

into its Dirichlet series. For $\operatorname{Re} s>1 / 2$, we have

$$
\begin{aligned}
& L\left(\frac{1}{2}+s+i r, g \otimes \chi\right) L\left(\frac{1}{2}+s-i r, g \otimes \chi\right) \\
= & \sum_{m, n \geq 1} \chi(m n) \frac{\lambda_{g}(m) \lambda_{g}(n)}{m^{1 / 2+s+i r} n^{1 / 2+s-i r}} .
\end{aligned}
$$

As $g$ is a Hecke eigenform, we have

$$
\lambda_{g}(m) \lambda_{g}(n)=\sum_{d \mid(m, n)} \lambda_{g}\left(\frac{m n}{d^{2}}\right) .
$$

Apply this to the right side of (2.5) and set $m=a d, n=b d$. Then

$$
\begin{aligned}
& L\left(\frac{1}{2}+s+i r, g \otimes \chi\right) L\left(\frac{1}{2}+s-i r, g \otimes \chi\right) \\
= & \sum_{a, b, d \geq 1} \chi\left(a b d^{2}\right) \frac{\lambda_{g}(a b)}{a^{1 / 2+s+i r} b^{1 / 2+s-i r} d^{1+2 s}} \\
= & L\left(1+2 s, \chi^{2}\right) \sum_{n \geq 1} \frac{\chi(n) \lambda_{g}(n)}{n^{1 / 2+s}} d_{i r}(n),
\end{aligned}
$$

where

$$
d_{s}(n)=\sum_{a b=|n|}\left(\frac{a}{b}\right)^{s} .
$$

We remark that in (2.6), the series is actually taken over $n$ which are relatively prime to $\mathcal{Q}$.

Consider the Eisenstein series for any fixed cusp $\mathfrak{a}$ of $\Gamma=\Gamma_{0}(\mathcal{N})$ defined by

$$
E_{\mathfrak{a}}(z, s)=\sum_{\gamma \in \Gamma_{\mathfrak{a}} \backslash \Gamma}\left(\operatorname{Im} \sigma_{\mathfrak{a}}^{-1} \gamma z\right)^{s}
$$

for Re $s>1$ and by analytic continuation for all $s \in \mathbb{C}$. Here $\Gamma_{\mathfrak{a}}$ is the stability group of $\mathfrak{a}$, while $\sigma_{\mathfrak{a}} \in S L(2, \mathbb{R})$ is given by $\sigma_{\mathfrak{a}} \infty=\mathfrak{a}$ and $\sigma_{\mathfrak{a}}^{-1} \Gamma_{\mathfrak{a}} \sigma_{\mathfrak{a}}=\Gamma_{\infty}$. This Eisenstein series is an eigenfunction of the Hecke operators

$$
T_{n} E_{\mathfrak{a}}(z, s)=\eta_{\mathfrak{a}}(n, s) E_{\mathfrak{a}}(z, s),
$$

if $(n, \mathcal{N})=1$. As pointed out in Conrey and Iwaniec [2], for any $n$ relatively prime to $\mathcal{N}, \eta_{\mathfrak{a}}(n, s)=d_{s-1 / 2}(n)$. Consequently, from (2.6) we get

$$
\begin{aligned}
& L\left(\frac{1}{2}+s+i r, g \otimes \chi\right) L\left(\frac{1}{2}+s-i r, g \otimes \chi\right) \\
= & L\left(1+2 s, \chi^{2}\right) \sum_{n \geq 1} \frac{\chi(n) \lambda_{g}(n) \eta_{\mathfrak{a}}(n, 1 / 2+i r)}{n^{1 / 2+s}}
\end{aligned}
$$

for $\operatorname{Re} s>1 / 2$. 
Substituting (2.7) into the integral of the main term in (2.4), we get

$$
\begin{aligned}
& L\left(\frac{1}{2}+i r, g \otimes \chi\right) L\left(\frac{1}{2}-i r, g \otimes \chi\right) \\
= & \frac{1}{\pi i} \int_{\operatorname{Re} s=2}\left(\sum_{m, n \geq 1} \frac{\chi\left(n m^{2}\right) \lambda_{g}(n) \eta_{\mathfrak{a}}(n, 1 / 2+i r)}{\left(n m^{2}\right)^{1 / 2+s}}\right) X^{s} G(s) \frac{d s}{s}+O\left(r^{\varepsilon}\right) \\
= & 2 \sum_{m, n \geq 1} \frac{\chi\left(n m^{2}\right) \lambda_{g}(n) \eta_{\mathfrak{a}}(n, 1 / 2+i r)}{m \sqrt{n}} \frac{1}{2 \pi i} \int_{\operatorname{Re}} G(s)\left(\frac{n m^{2}}{X}\right)^{-s} \frac{d s}{s}+O\left(r^{\varepsilon}\right) .
\end{aligned}
$$

Denote

$$
V(y)=\frac{1}{2 \pi i} \int_{\operatorname{Re} s=2} G(s) y^{-s} \frac{d s}{s} .
$$

Then as in [22] and [18, $\lim _{y \rightarrow 0} V(y)=1$ and $V(y) \ll_{B}(1+|y|)^{-B}$ because of our choice of the function $G(s)$. Therefore

$$
\begin{aligned}
& L\left(\frac{1}{2}+i r, g \otimes \chi\right) L\left(\frac{1}{2}-i r, g \otimes \chi\right) \\
= & 2 \sum_{\substack{1 \leq m \leq X^{1 / 2+\varepsilon}\\
}} \frac{\chi^{2}(m)}{m} \sum_{n \geq 1} \frac{\chi(n) \lambda_{g}(n) \eta_{\mathfrak{a}}(n, 1 / 2+i r)}{\sqrt{n}} V\left(\frac{n m^{2}}{X}\right),
\end{aligned}
$$

because the outer series is negligible if taken over $m>X^{1 / 2+\varepsilon}$.

\section{Averaging and the Kuznetsov trace formula}

According to (2.8), estimation of the central value of our $L$-function is reduced to estimation of

$$
S_{Y}(g, r)=\sum_{n} \chi(n) \lambda_{g}(n) \eta_{\mathfrak{a}}(n, 1 / 2+i r) H\left(\frac{n}{Y}\right)
$$

for fixed $g$, where $H$ is a fixed smooth function of compact support contained in $(1,2)$.

To prove our results on short intervals, let $L$ be a number which satisfies $\sqrt{K} \leq$ $L \leq K / 4$ for large $K$. Let $h(t)$ be an even analytic function in $|\operatorname{Im} t| \leq 1 / 2$ satisfying $h^{(n)}(t) \ll(1+|t|)^{-N}$ for any $N>0$ in this region. Thus $h$ is a Schwartz function on $\mathbb{R}$. We also assume that $h(t) \geq 0$ for real $t$. For example, we may simply take $h(t)=1 / \cosh (t)$. Denote

$$
\zeta_{\mathcal{N}}(s)=\prod_{p \mid \mathcal{N}}\left(1-p^{-s}\right)^{-1}
$$


We want to estimate

$$
\begin{aligned}
I_{K, L}= & \int_{\mathbb{R}}\left(h\left(\frac{K-r}{L}\right)+h\left(\frac{K+r}{L}\right)\right)\left|S_{Y}(g, r)\right|^{2} \frac{\left|\zeta_{\mathcal{N}}(1+2 i r)\right|^{2}}{|\zeta(1+2 i r)|^{2}} d r \\
= & \sum_{m, n} \chi(n) \bar{\chi}(m) \lambda_{g}(n) \bar{\lambda}_{g}(m) H\left(\frac{n}{Y}\right) \bar{H}\left(\frac{m}{Y}\right) \\
& \times \int_{\mathbb{R}}\left(h\left(\frac{K-r}{L}\right)+h\left(\frac{K+r}{L}\right)\right) \\
& \times \eta_{\mathfrak{a}}(n, 1 / 2+i r) \bar{\eta}_{\mathfrak{a}}(m, 1 / 2+i r) \frac{\left|\zeta_{\mathcal{N}}(1+2 i r)\right|^{2}}{|\zeta(1+2 i r)|^{2}} d r .
\end{aligned}
$$

As in Liu and Ye [18, we apply the Kuznetsov trace formula to the integral on the right side of (3.1):

$$
\begin{aligned}
& \pi \sum_{m, n} \chi(n) \bar{\chi}(m) \lambda_{g}(n) \bar{\lambda}_{g}(m) H\left(\frac{n}{Y}\right) \bar{H}\left(\frac{m}{Y}\right) \\
& \times \sum_{f_{j}}\left(h\left(\frac{K-k_{j}}{L}\right)+h\left(\frac{K+k_{j}}{L}\right)\right) \lambda_{f_{j}}(n) \bar{\lambda}_{f_{j}}(m) \\
+ & \sum_{m, n} \chi(n) \bar{\chi}(m) \lambda_{g}(n) \bar{\lambda}_{g}(m) H\left(\frac{n}{Y}\right) \bar{H}\left(\frac{m}{Y}\right) \\
& \times \int_{\mathbb{R}}\left(h\left(\frac{K-r}{L}\right)+h\left(\frac{K+r}{L}\right)\right) \\
& \times \eta_{\mathfrak{a}}(n, 1 / 2+i r) \bar{\eta}_{\mathfrak{a}}(m, 1 / 2+i r) \frac{\left|\zeta_{\mathcal{N}}(1+2 i r)\right|^{2}}{|\zeta(1+2 i r)|^{2}} d r \\
= & \sum_{m, n} \chi(n) \bar{\chi}(m) \lambda_{g}(n) \bar{\lambda}_{g}(m) H\left(\frac{n}{Y}\right) \bar{H}\left(\frac{m}{Y}\right) \\
& \times \frac{\delta_{n, m}}{\pi} \int_{\mathbb{R}} \tanh (\pi r)\left(h\left(\frac{K-r}{L}\right)+h\left(\frac{K+r}{L}\right)\right) r d r \\
+ & 2 i \sum_{m, n} \chi(n) \bar{\chi}(m) \lambda_{g}(n) \bar{\lambda}_{g}(m) H\left(\frac{n}{Y}\right) \bar{H}\left(\frac{m}{Y}\right) \\
& \times \sum_{c \geq 1} \frac{S(n, m ; c)}{c} \int_{\mathbb{R}} J_{2 i r}\left(\frac{4 \pi \sqrt{n m}}{c}\right) \\
& \times\left(h\left(\frac{K-r}{L}\right)+h\left(\frac{K+r}{L}\right)\right) \frac{r d r}{\cosh (\pi r)} .
\end{aligned}
$$

Here in (3.2) $f_{j}$ are Hecke eigenforms, with Laplace eigenvalues $1 / 4+k_{j}^{2}$ and Fourier coefficients $\lambda_{f_{j}}(n)$, which form an orthonormal basis of the space of Maass cusp forms for $\Gamma_{0}(\mathcal{N})$, while in (3.5) $S(n, m ; c)$ is the classical Kloosterman sum.

Recall that $\chi(n) \lambda_{g}(n)$ is the $n$th Fourier coefficient of the twisted cusp form $g_{\chi}$ as in (2.1) or (2.2). We want to apply the main estimation in Liu and Ye 18 $(\S 4.1-\$ 4.13)$ and Lau, Liu, and Ye [17, (2.2), to our (3.4) and (3.5) above. Note that these estimations are based on bounds for shifted convolution sums of Fourier 
coefficients of cusp forms proved by Sarnak 22, Appendix, and by Lau, Liu, and Ye [17.

More precisely, $(3.4)+(3.5) \ll L K Y^{1+\varepsilon}$ for $L=K^{1-1 /(4+2 \theta)+\varepsilon}$. Since (3.2) and (3.3) are both positive, this implies that (3.3), i.e., $I_{K, L}$, is bounded by $O\left(L K Y^{1+\varepsilon}\right)$ for the same $L$. By $\zeta(1+2 i r) \ll \log (1+|r|)$, this estimate of $I_{K, L}$ implies that

$$
\int_{K}^{K+L}\left|S_{Y}(g, r)\right|^{2} d r \ll L K Y^{1+\varepsilon}
$$

for the above $L$.

Now we can go back to the fourth power moment of $L(1 / 2+i r, g \otimes \chi)$. Since $g$ is self-contragredient and $\chi$ is real, we have from (2.8) that

$$
\begin{aligned}
& \int_{K}^{K+L}\left|L\left(\frac{1}{2}+i r, g \otimes \chi\right)\right|^{4} d r=\int_{K}^{K+L}\left|L\left(\frac{1}{2}+i r, g \otimes \chi\right) L\left(\frac{1}{2}+i r, g \otimes \chi\right)\right|^{2} d r \\
\ll & \int_{K}^{K+L}\left|\sum_{1 \leq m \leq X^{1 / 2+\varepsilon}} \frac{\chi^{2}(m)}{m} \sum_{n \geq 1} \frac{\chi(n) \lambda_{g}(n) \eta_{\mathfrak{a}}(n, 1 / 2+i r)}{\sqrt{n}} V\left(\frac{n m^{2}}{X}\right)\right|^{2} d r .
\end{aligned}
$$

Here we can take $X=K^{2}$ and get

$$
\begin{aligned}
& \int_{K}^{K+L}\left|L\left(\frac{1}{2}+i r, g \otimes \chi\right)\right|^{4} d r \\
\ll & \frac{1}{K^{2}} \int_{K}^{K+L}\left|\sum_{n \geq 1} \chi(n) \lambda_{g}(n) \eta_{\mathfrak{a}}(n, 1 / 2+i r) \sum_{1 \leq m \leq K^{1+\varepsilon}} \chi^{2}(m) \frac{V\left(n m^{2} / K^{2}\right)}{\sqrt{n m^{2} / K^{2}}}\right|^{2} d r .
\end{aligned}
$$

Now we apply a smooth dyadic subdivision to

$$
\sum_{1 \leq m \leq K^{1+\varepsilon}} \chi^{2}(m) \frac{V\left(n m^{2} / K^{2}\right)}{\sqrt{n m^{2} / K^{2}}},
$$

by dividing the interval $\left[1, K^{1+\varepsilon}\right]$ into subintervals of the form $[a, 1.8 a]$ and covering, with overlapping, each subinterval by a smooth, nonnegative function of compact support. The total number of subintervals is $O(\log K)$. This way, we can find a smooth function $H$ of compact support in $(1,2)$ so that

$$
\begin{aligned}
\int_{K}^{K+L}\left|L\left(\frac{1}{2}+i r, g \otimes \chi\right)\right|^{4} d r \\
\ll \frac{\log K}{K^{2}} \int_{K}^{K+L} \max _{1 \leq B \leq K^{2+\varepsilon}}\left|\sum_{n \geq 1} \chi(n) \lambda_{g}(n) \eta_{\mathfrak{a}}(n, 1 / 2+i r) H\left(\frac{n}{K^{2} / B}\right)\right|^{2} d r .
\end{aligned}
$$


The sum inside the absolute value signs is indeed $S_{K^{2} / B}(g, r)$. By (3.6), the maximum contribution is from $B=1$ :

$$
\begin{aligned}
\int_{K}^{K+L}\left|L\left(\frac{1}{2}+i r, g \otimes \chi\right)\right|^{4} d r & \ll \frac{\log K}{K^{2}} \int_{K}^{K+L}\left|S_{K^{2}}(g, r)\right|^{2} d r \\
& \ll \frac{\log K}{K^{2}} L K\left(K^{2}\right)^{1+\varepsilon} \ll(K L)^{1+\varepsilon}
\end{aligned}
$$

for $L=K^{1-1 /(4+2 \theta)+\varepsilon}$. This completes the proof of Theorem 1.1.

\section{ADDED IN PROOF}

Recently, Lau, Liu, and Ye further improved the subconvexity bound (1.4) to $k^{3 / 4+\varepsilon}$. Using this new result, our Theorem 1.1 can be stated for $L=K^{1 / 2+\varepsilon}$.

\section{REFERENCES}

[1] A.O.L. Atkin and Wen-Ch'ing W. Li, Twists of newforms and pseudo-eigenvalues of Woperators, Invent. Math., 48 (1978), 221-243. MR0508986 (80a:10040)

[2] L.B. Conrey and H. Iwaniec, The cubic moment of central values of automorphic L-functions, Ann. Math., 151 (2000), 1175-1216. MR1779567 (2001g:11070)

[3] W. Duke, J.B. Friedlander, and H. Iwaniec, Bounds for automorphic L-functions, Invent. Math., 112 (1993), 1-8. MR.1207474 (94c:11043)

[4] W. Duke, J.B. Friedlander, and H. Iwaniec, Bounds for automorphic L-functions II, Invent. Math., 115 (1994), 219-239; erratum, ibid. 140 (2000), 227-242. MR1258904 (95a:11044)

[5] W. Duke, J.B. Friedlander, and H. Iwaniec, Bounds for automorphic L-functions III, Invent. Math., 143 (2001), 221-248. MR1835388(2003c:11043)

[6] A. Good, Beiträge zur Theorie der Dirichletreihen, die Spitzenformen zugeordnet sind, J. Number Theory, 13 (1981), 18-65. MR0602447 (82i:10034)

[7] A. Good, The square mean of Dirichlet series associated with cusp forms, Mathematika, 29 (1982), 278-295. MR0696884 (84f:10036)

[8] A. Good, The convolution method for Dirichlet series, in: D. Hejhal, P. Sarnak, A. Terras (Eds.), The Selberg trace formula and related topics (Brunswick, Maine, 1984), Amer. Math. Soc. Contemporary Mathematics, Vol. 53, Amer. Math. Soc., Provindence, RI, 1986, 207-214. MR0853560 (87m:11047)

[9] D.R. Heath-Brown, The fourth power moment of the Riemann zeta-function, Proc. London Math. Soc., 38 (1979), 385-422. MR0532980 (81f:10052)

[10] A. Ivic, The Riemann zeta-function. The theory of the Riemann zeta-function with applications, John Wiley \& Sons, New York, 1985. MR0792089(87d:11062)

[11] H. Iwaniec, Fourier coefficients of cusp forms and the Riemann zeta function, Séminaire de Théorie des Nombres de Bordeaux, Année 1979-1980, exposé $n^{\circ}$ 18, 1-36. MR0604215 (82i:10047)

[12] H. Kim, Functoriality for the exterior square of $G L_{4}$ and the symmetric fourth of $G L_{2}$, J. Amer. Math. Soc., 16 (2003), 139-183. MR1937203 (2003k:11083)

[13] H. Kim and P. Sarnak, Appendix: Refined estimates towards the Ramanujan and Selberg conjectures, Appendix to Kim [12]. MR1937203 (2003k:11083)

[14] H. Kim and F. Shahidi, Cuspidality of symmetric powers with applications, Duke Math. J., 112 (2002), 177-197. MR 1890650 (2003a:11057)

[15] E. Kowalski, P. Michel, and J. Vanderkam, Mollification of the fourth moment of automorphic L-functions and arithmetic applications, Invent. Math., 142 (2000), 95-151. MR 1784797 (2001m:11080)

[16] N.V. Kuznetsov, Petersson's conjecture for cusp forms of weight zero and Linnik's conjecture. Sums of Kloosterman sums, Math. U.S.S.R. Sbornik, 29 (1981), 299-342. MR0568983 (81m:10053)

[17] Yuk-Kam Lau, Jianya Liu, and Yangbo Ye, Subconvexity bounds for Rankin-Selberg Lfunctions for congruence subgroups, to appear in J. Number Theory. 
[18] Jianya Liu and Yangbo Ye, Subconvexity for Rankin-Selberg L-functions of Maass forms, Geom. Funct. Anal., 12 (2002), 1296-1323. MR.1952930 (2004b:11060)

[19] Jianya Liu and Yangbo Ye, Petersson and Kuznetsov trace formulas, in Lie Groups and Automorphic Forms, Amer. Math. Soc. and International Press, 2005.

[20] W. Luo, Z. Rudnick, and P. Sarnak, On the generalized Ramanujan conjecture for $G L(n)$, in: Automorphic forms, automorphic representations, and arithmetic (Fort Worth, TX, 1996), 301-310, Proc. Sympos. Pure Math., 66, Part 2, Amer. Math. Soc., Providence, RI, 1999. MR.1703764 (2000e:11072)

[21] T. Meurman, On the order of the Maass L-function on the critical line, in: K. Györy, G. Halász (Eds.), Number Theory, Vol. I (Budapest, 1987), Colloquium of Mathematical Society Janos Bolyai, Vol. 51, North-Holland, Amsterdam, 1990, 325-354. MR1058223 (91m:11037)

[22] P. Sarnak, Estimates for Rankin-Selberg L-functions and quantum unique ergodicity, J. Functional Analysis, 184 (2001), 419-453. MR1851004 (2003c:11050)

[23] F. Shahidi, On the Ramanujan conjecture and finiteness of poles for certain L-functions, Ann. of Math., (2) 127 (1988), no. 3, 547-584. MR0942520 (89h:11021)

Department of Mathematics, The University of Iowa, Iowa City, Iowa 52242-1419

E-mail address: yey@math.uiowa.edu 